\title{
10.22402/j.rdipycs.unam.7.2.2021.357.287-305
}

\section{EL PENSAMIENTO DE TERCER ORDEN EN LA TERAPIA BREVE ESTRATÉGICA PARA PAREJAS QUE VIVIERON UNA INFIDELIDAD}

\author{
Mario Fausto Gómez Lamont \\ Facultad de Estudios Superiores Iztacala, UNAM \\ México
}

\begin{abstract}
RESUMEN
El pensamiento de tercer orden es promotor de reflexiones acerca de las desigualdades sociales como ejes rectores de las hipótesis de trabajo de las(os) terapeutas. Diversos autores(as) han referido a la terapia con enfoque estratégico como un método breve, eficaz y eficiente para el trabajo con las parejas que - mediante el pensamiento de tercer orden, la circularidad, las soluciones intentadas y las jerarquías incongruentes-dan un giro de 180 grados, pues el/la terapeuta es un agente del cambio social. En particular por su capacidad de analizar los patrones interaccionales en la conducta de las parejas desde la comprensión de cómo funciona la cultura y el poder, que se ven reflejados en los síntomas como formas de resistencia cotidiana. La terapia breve estratégica es un método capaz de resolver en poco tiempo las conductas problemáticas en las parejas. Hablando de la infidelidad, la terapia estratégica ofrece elementos resolutos de este problema. En este escrito se usó un análisis conceptual del modelo estratégico para el trabajo con parejas heterosexuales, lo que permitió reflexionar acerca de la metodología y la ética de usarlo en otro tipo de parejas.
\end{abstract}

Palabras Clave:

pensamiento de tercer orden, terapia breve estratégica, parejas

\section{THIRD-ORDER THINKING IN BRIEF STRATEGIC THERAPY FOR COUPLES WHO HAVE EXPERIENCED INFIDELITY}

\begin{abstract}
Third-order thinking is a promoter of reflections on social inequalities as guiding axes of the therapists' working hypotheses. Various authors have referred to therapy with a strategic approach as a brief, effective and efficient method for working with couples, which through third-order thinking, circularity, attempted solutions and incongruous hierarchies give a 180 turn degrees, since the therapist is an agent of social change. Particularly for its ability to analyze interactional patterns in the behavior of couples from the understanding of how culture and power operate, which are reflected in the symptoms as forms of daily resistance. Brief strategic therapy is a method capable of solving problem behaviors in couples in a short time. Speaking specifically of infidelity, strategic therapy provides solving elements of this problem. In this writing, a conceptual analysis of the strategic model for working with heterosexual couples was used, which led us to reflect on the methodology and ethics of using it in other types of couples.
\end{abstract}

Keywords:

third order thinking, brief strategic therapy, couples

Bitácora del Artículo:

| Recibido: 26 de Marzo de 2021 | Aceptado: 29 de Junio de 2021 | Publicado en línea: Julio-Diciembre de 2021 | 
Artículo teórico | El pensamiento de tercer orden en la terapia Greve estratégica para parejas...| Gómez-Lamont

Autoría y Derechos de Propiedad Intelectual

\title{
EL PENSAMIENTO DE TERCER ORDEN EN LA TERAPIA BREVE ESTRATÉGICA \\ PARA PAREJAS QUE VIVIERON UNA INFIDELIDAD
}

\author{
Mario Fausto Gómez Lamont \\ Facultad de Estudios Superiores Iztacala, UNAM \\ México
}

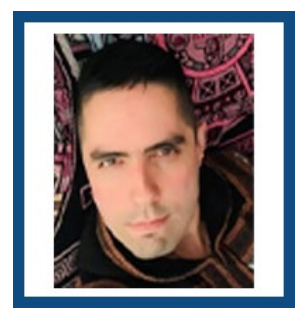

Mario Fausto Gómez Lamont

FES- Iztacala, UNAM

Correo: fausto.lamont@iztacala.unam.mx

\begin{abstract}
Especialista y maestro en Medicina Social por la Universidad Autónoma Metropolitana campus Xochimilco. Maestro en Psicoterapia Familiar Sistémica por la Universidad Del Valle de México. Ha sido consultor para la Organización de las Naciones Unidas (ONU-Mujeres) y para el Consejo Nacional para Prevenir y Erradicar la Violencia contra las Mujeres (CONAVIM) formando parte del grupo de trabajo para la activación de la alerta de género en el estado de Puebla. Tuvo intervenciones en el Senado de la República acompañando la iniciativa de Ley contra las Terapias de Conversión. Actualmente es profesor definitivo en la FES Iztacala de la UNAM, así como profesor invitado en el posgrado de Medicina Familiar de la Facultad de Medicina de la UNAM.
\end{abstract}

\section{CONTRIBUCIÓN DE LOS AUTORES}

Se atribuye al autor la responsabilidad única sobre el trabajar al concibir, desarrollar y coordinar el proyecto de investigación.

\section{AGRADECIMIENTOS}

Quiero agradecer a la Dra. J. María Bermudez por sus enseñanzas y confianza absoluta en mi escritura y mi forma de pensar en tercer orden.

\section{Datos de Filiación de los Autores}

Facultad de Estudios Superiores Iztacala, UNAM

\section{(c) (i) (2) (2)}

Copyright: (c) 2021 Gómez-Lamont, M.F.

Este es un artículo de acceso abierto distribuido bajo los términos de la licencia Creative Commons Reconocimiento-NoComercial 4.0 Internacional, por lo que su contenido gráfico y escrito se puede compartir, copiar y redistribuir total o parcialmente sin necesidad de permiso expreso de sus autoras con la única condición de que no se puede usar con fines directamente comerciales y los términos legales de cualquier trabajo derivado deben ser los mismos que se expresan en la presente declaración. La única condición es que se cite la fuente con referencia a la Revista Digital Internacional de Psicología y Ciencia Social y a sus autoras. 


\section{TABLA DE CONTENIDO}

INTRODUCCIÓN

ANTECEDENTES CONCEPTUALES

PRECISIONES CONCEPTUALES

ESTRATEGIAS PARA LA TERAPIA DE PAREJA

CORRELACIONES TEÓRICAS

Conocer la historia de amor de la pareja, 293

Formular un enfoque estratégico, 294

Formular tácticas concretas, 294

Encuadrar la sugerencia en un contexto: Vender la tarea, 295

Formular objetivos y evaluar el resultado, 295

ANÁLISIS Y DISCUSIÓN 


\section{INTRODUCCIÓN}

$\mathbf{P}$ ara McDowell, Kudson-Martin y Bermudez (2019) el pensamiento de tercer orden contribuye a que las(os) terapeutas inviten a sus consultantes a considerar el punto de vista del problema de cada uno de los(as) integrantes de la familia o pareja, desde las dinámicas de la dominación, subordinación y discriminación sociocultural expresadas en síntomas o metáforas asociadas al problema. En el proceso se escuchan las voces de quienes están en posiciones inferiores e intervienen en jerarquías incongruentes al visibilizar esas redes de subordinación que se encuentran invisibles. Se anima a las parejas a visualizar e intentar nuevas formas de relación que conduzcan a una transformación de sus relaciones para que no estén limitadas por lo socialmente dicho, como los roles de género, la heterosexualidad obligatoria, el clasismo y racismo, entre otros sistemas de opresión y subordinación presentes en la cultura.

Los patrones interaccionales son categorías analíticas que servirán al (la) terapeuta que se adscribe con estratégico, familiar y de pareja, para producir cambios ante los problemas que presentan las familias y parejas (Madanes, 2007). Es así como me gustaría reconocer en este escrito tres objetivos: 1) describir el pensamiento de tercer orden en la terapia breve estratégica para parejas; 2) analizar las formas particulares de trabajar un caso ficticio de una pareja heterosexual mediante este modelo (Madanes, 2007; Vicencio, 2011; Fisch, Weakland y Segal, 2003), y 3) visibilizar las problemáticas éticas de la terapia estratégica para fundamentar la necesidad de integrar el pensamiento de tercer orden a este tipo de terapia (Gutiérrez, 2018; Gómez-Lamont, 2016; Butler, 2015).

\section{Antecedentes Conceptuales}

Se puede apreciar a la pareja heterosexual como un grupo instituido en la sociedad humana (Vicencio, 2011). De igual modo, como todo grupo instituido se conforma de un arreglo ficticio y litúrgico que se validará sobre otras formas de expresión de pareja (lo que conocemos como heteronorma). En este sentido a la pareja heteronormada se le puede considerar como "legítima" en la sociedad si, y sólo si, cumple con el imaginario social, el cual implica compromiso, amor, estabilidad y deseos de "fundar una familia" (Roizblatt, 2006). Porque per- tenecer a un grupo implica demostrar una creencia en los mitos y en las ficciones de ese grupo de pertenencia, así como la participación en los rituales de la familia de origen de cada uno(a) de los(as) integrantes de la pareja. Esta pertenencia exige más que palabras (Sternberg, 1998). Para Madanes (2007), ante las exigencias del exterior las parejas suelen resolver las luchas mediante síntomas para equilibrar el poder en la relación.

En ese sentido, Neuburger (1995) indica que pertenecer a una pareja tendrá un costo porque las parejas suelen ejercer un poder no sólo con la posibilidad de dominar al cónyuge sino también de "reconfortarlo(a)", cuidarlo(a), reformarlo(a) y asumir, quizá, responsabilidad por él (ella) (Madanes, 2007). En este sentido se observa una solidaridad frente a las intrusiones del mundo exterior, fronteras que se presentarán en límites, sean éstos rígidos o flexibles. Pero recordando los costos, la solidaridad y protección actuará mientras el sujeto interesado se muestre fiel al grupo. Esta misma solidaridad puede actuar contra el sujeto si sale a la luz de la relación alguna infidelidad (Madanes, 2007).

Pero para hablar de infidelidad, es fundamental definir el término "fidelidad", el cual proviene del latín fidelitas-atis y significa "lealtad", "cumplida adhesión", "conservación de la fe en el cumplimiento de la sinceridad", así como el cumplimiento de la expresión de los afectos y promesas, por ello se muestra digno de confianza (Baizán, 2007).

Un tema relacionado con la fidelidad es el de intimidad, la cual se construye de modo gradual a medida que crece esta confianza y fe en la relación, donde, a pesar de las diferencias encontradas entre los que conforman a la pareja, sigue habiendo el aprecio, respeto y afecto por lo que es del otro (Baizán, 2007).

Entonces, sin compromiso ni fidelidad la intimidad y la cercanía mostrarían y darían a sospechar sobre una base insegura a los miembros que quisieran formalizar la relación, en la cual el aventurarse podría ser peligroso para la subsistencia física y emocional de cualquiera de los(as) integrantes (Vicencio, 2011).

Recordando así que quién rete al grupo de pertenencia mediante la infidelidad se considera que será responsabilidad de quien decide involucrarse en dicha relación, por lo que uno de los grandes problemas en esta situación es la revelación de que el (la) integrante no se encuentra cómodo(a), que juzga que el grupo no le aporta lo que él (ella) espera o que no puede aceptar su evolución. En estas situaciones la pertenencia a otra pareja a menudo será la causa más seductora para el (ella) y aparecerá como un(a) rival peligroso(a) para la pareja en turno, por lo que, revelada la infidelidad, 
marca al (a la) infiel como el (la) extranjero(a) en la relación traicionada. Es así como pierde toda protección y se expone, según el caso, a violencias cuyo objetivo puede ser traerlo(a) al seno de la relación anterior, o excluirlo(a) de manera definitiva.

La infidelidad puede ser vista como un intento por resolver un problema personal, algo que ocurre dentro de la persona que no puede ser solucionado o satisfecho con su pareja. En ese sentido Madanes (2007) piensa que la infidelidad puede ser una conducta sintomática de uno de los cónyuges que busca, de algún modo, cambiar la conducta del otro cónyuge. Esta conducta sintomática es una solución desafortunada porque en vez de equilibrar la situación produce una incongruencia en la relación. Desde el pensamiento de tercer orden (McDowell et al., 2019) la infidelidad tendrá significados diferentes, y éstos dependerán del género, la orientación sexual, la clase social, la zona geográfica y la religión, pues hombres y mujeres son infieles de manera diferenciada, donde en los primeros es más común que en las segundas (muchas veces hasta son justificados socialmente); en parejas de hombres homosexuales la infidelidad tiene una connotación más acentuada en la separación del amor y el placer, pues ésta puede llegar a ser consensuada y en algunos casos no se nombra ya como infidelidad, al igual que en el caso de mujeres homosexuales infieles, que después del descubrimiento pueden significarlo de modos diversos y constructivos para la relación, o la separación definitiva (Gómez-Lamont y Silva, 2019).

\section{Precisiones conceptuales}

Algunas hipótesis comunes en el espacio terapéutico, como las de Asen y Jones (2000), sugieren en pensar en síntomas depresivos que puedan estar presentes en las parejas después la infidelidad; sin embargo, y retomando las ideas de tercer orden, será fundamental incluir otras hipótesis de trabajo con tal de evitar la patologización de las situaciones relacionales (Mcdowell et al., 2019).

Las bases de la terapia breve estratégica mediante el pensamiento de tercer orden incluyen las siguientes hipótesis de trabajo.

- Los problemas como soluciones intentadas. Desde esta perspectiva los problemas son síntomas que determinan una comprensión a profundidad, e incluye el sistema más amplio, es decir, el contexto social: la infidelidad deberá ser vista como parte del sistema de creencias con base en el género, la necesidad de satisfacer los impulsos sexuales de los hombres, la hipersexualización de las mujeres, la pornografía y el tráfico de mujeres. Este sistema de creencias falocentrista se nutre de símbolos imaginarios, configurando subjetividades que brincan las orientaciones sexuales, es decir, que no es exclusiva de la heterosexualidad, sino que puede ser inteligible en las relaciones homosexuales y bisexuales, haciendo del deseo sexual una necesidad y una búsqueda de la satisfacción del deseo por medio del consumo de cuerpos estereotipados por el mercado (Gómez-Lamont y Silva, 2019).

- Pensamiento contraintuitivo (o no lineal). Para McDowell et al. (2018) una de las premisas básicas es el pensamiento circular en la terapia breve y estratégica; éste permite ser contraintuitivo o no lineal, con tal de ser capaz de observar los cambios de primer orden (superficiales) con los de segundo orden (profundos y estructurales); sin embargo, y en palabras de ellas, no es suficiente pues es necesario "Ser capaz de adoptar una metavisión de los discursos sociales dominantes, y la dinámica de los sistemas sociales amplios permite a los terapeutas desarrollar la conciencia crítica necesaria para pensar tanto de forma contrahegemónica como contraintuitiva. Usamos el término hegemonía para referirnos a los mecanismos que mantienen el statu quo de distribución desigual del poder en la sociedad a través del control social, político, económico e ideológico. Esto incluye, pero va más allá, el concepto de discursos dominantes para incluir leyes, prácticas corporativas y educativas, servicios sociales, etc. (pp. 67)".

- Jerarquías incongruentes. Las jerarquías incongruentes dejan de tener significados normativos en términos de subordinación de las mujeres o de quien exprese feminidad en la relación hetero, homo, bi y trans; para el pensamiento de tercer orden, una jerarquía incongruente es la que no permite la igualdad y la equidad de la distribución de las condiciones materiales y emocionales de su cotidianidad.

Baizán (2007) coincide con el pensamiento de tercer orden (McDowell et al., 2018) porque considera que las creencias y el juicio que se incorporan en la infidelidad, ejerce una influencia importante en el enfoque y la estrategia de abordaje de una pareja ante esta crisis ocasionada por la infidelidad (Asen y Jones, 2000). Mediante la presentación de un caso se propondrá una forma de abordaje terapéutico desde el enfoque sistémico, precisando en el modelo de terapia breve estratégica. 


\section{Estrategias para la terapia de PAREJA}

Este enfoque de terapia breve fue creado en torno a 1968, poco después de la muerte de Don D. Jackson, quien fundó el grupo del Mental Research Institute (MRI) en 1959. Quienes se quedaron a cargo de este grupo de investigación fueron Watzlawick, Weaklan, Fish y Segal. Donde la forma de proceder del grupo del MRI se encuentra caracterizado por hacer intervenciones en todas las respuestas o intentos de solución que los pacientes, parejas y familias elaboran para solucionar la situación, dificultad o problema que genera el síntoma y que por diversas circunstancias estos intentos de solución han resultado insatisfactorios.

Por otra parte Vicencio (2011) reconoce que uno de los principales motivos de consulta para un terapeuta de pareja es la vida amorosa, por lo que recuerda que Fisch, Weakland y Segal (2003) aportan a la terapia de pareja el análisis de patrones recurrentes que definen las actuaciones de los(as) participantes por encima de las características individuales. Siguiendo esa línea de pensamiento, Madanes (2007) recuerda que la interacción de las parejas respecto a la infidelidad se convertirá en una metáfora de su interacción en torno a otros problemas, es decir, que la desconfianza será la metáfora resultante de la infidelidad y, de manera cíclica, se verá aplicada a otros problemas.

Los(as) autores(as) afirman que si se concibe a la psicoterapia como un proceso donde el (la) terapeuta funge como un(a) promotor(a) del cambio, las herramientas que debiese llegar a utilizar deberán marcar en manera oportuna las oportunidades positivas que tiene la pareja de hacer algo diferente o distinto a lo que viene haciendo desde hace un buen tiempo, motivo que convierte a una dificultad en un problema. Para ello, tener una estructura sólida de la concepción general de los problemas y el tratamiento, estructurados en un conocimiento de la teoría expuesta por los(as) autores(as), se vincularán a las prácticas concretas de este enfoque terapéutico, donde por medio de la reflexión se aceptará el uso o aplicación de éstas. Es así como McDowell et al. (2018) consideran que las(os) terapeutas breves y estratégicos(as) que piensan en tercer orden tienen la tarea de comprender cómo las interacciones de las parejas y el significado de los problemas son afectados por la cultura y la dinámica social de poder y subordinación.

Por ejemplo, Vicencio (2011) recuerda los juegos interaccionales que las parejas crean en su muy particular construcción del amor; éstos pueden ser: "Yo estoy bien tú estás mal", "Gana siempre el que dice la última palabra", "El que se enoja pierde", y "Qué sería de mí sin él". Estos juegos interaccionales son parte de los "lentes" que todo(a) terapeuta deberá reconocer en una pareja - a sugerencia del autor-, con tal de fomentar el cambio, rompiendo los patrones interaccionales que sostienen a la pareja en un estado de sufrimiento. A su vez, esta propuesta puede nutrirse de las hipótesis acerca del género; por ejemplo, es más común encontrar en los hombres un juego interaccional relacionado con el "Yo estoy bien y tú estás mal", "Gana siempre el que dice la última palabra", o "El que se enoja pierde", dada la cultura al éxito enfocada en las figuras masculina y femenina "Qué sería de mí sin él".

Para Vicencio (2011) es importante tener una perspectiva amplia y profunda de los conflictos de las parejas, por lo que nos recuerda lo que Gottman (1994) propone respecto a los crecientes problemas en las parejas: "Los cuatro jinetes del Apocalipsis"; Gottman (1994) apuesta a mirar otros patrones negativos y redundantes en las parejas; dichos patrones se pueden diferenciar en: 1) críticas dirigidas a los defectos del otro como persona; 2) desprecio y sarcasmo, actitudes defensivas que incluyen irritabilidad y agresión, y 3) actitudes evasivas que levantan una pared de hielo en la pareja. Para Gottman (1994) los patrones interaccionales negativos pueden predecir en $96 \%$ el divorcio o el rompimiento de la pareja. Un aporte del pensamiento de tercer orden (McDowell et al., 2019) es observar cómo son los cruces entre el género, la orientación sexual, la clase social y condición geográfica relacionadas a estas afirmaciones, pues, en un caso de hombres homosexuales donde uno de ellos reconozca sus orígenes indígenas y que debido al racismo en su pareja pudieran presentarse "las críticas dirigidas a los defectos del otro como persona" relacionadas a su tono de piel o creencias de su familia de origen. En una pareja heterosexual donde la mujer gane más dinero que el hombre las "actitudes evasivas que levantan una pared de hielo entre la pareja" pudieran afectar al hombre en su relación con su compañera debido al clasismo y el sexismo cultural que han imposibilitado el diálogo entre ellos, pues se considera que los hombres deberán mantener a sus esposas; por tanto deberán ganar más que ellas, consiguiendo con ello, un malestar en su relación.

Para Sternberg (1998) estos patrones pueden mirarse en las historias que conforman a las parejas; en esa interacción e intimidad conforman una forma particular de amarse. Esta historia no es intacta; el autor describe que se va modificando remplazando historias nuevas por viejas. Para Sternberg (1998) las historias de amor tienen guiones, tramas y actores. Los guiones son tramas resumidas de lo que ocurre en la relación. La trama puede ser vista como una serie de eventos en las que están implicados el amor, la confianza y los cuidados, que son interpretados por los(as) 
integrantes de la pareja que dan sentido a la relación. En cuanto a los actores, son quienes integran la pareja y los implicados en esa historia; es importante reconocer cuál es el tipo de historia de amor cruzada y entretejida entre estos tres elementos, actores, tramas y guiones. Estas historias pueden visibilizar los contextos culturales en que fueron criados ambos integrantes de la familia. Desde el pensamiento de tercer orden (McDowell et al., 2018, 2019) se explorarían en la historia de la pareja las condiciones de atracción y rechazo; por ejemplo, si le atraía que su pareja fuera exitosa(o), que tuviera un color de piel específico, un cuerpo específico, estudios universitarios, una orientación sexual o identidad de género específico; también se indagaría — con quienes nunca se hubieran relacionado- cuál sería su ideal en cuestión de estatus económico de pareja y la impresión de la familia ante alguna pareja que no fuera parte de la expectativa, entre otras condiciones sociales que son parte de la trama e historia de amor.

Siguiendo las historias de amor, Madanes (2007), en el trabajo con parejas, relaciona con la idea de que el terapeuta redefina el motivo de consulta de la pareja: de algo aislado a algo propio y - en palabras de Sternberg - parte de la historia de amor de la pareja; en ese tenor la autora propone al o la terapeuta ir conectando la idea de interacción en torno al síntoma; en ese sentido McDowell et al. (2018, 2019) se unen a la propuesta: la redefinición del motivo de consulta no sólo debe de conectar al integrante con la pareja, sino a la pareja con el sistema social.

Por último, pero no menos importante, en la propuesta que hace Vicencio (2011) de los mapas del amor, nos recuerda a Díaz-Loving (2005), que propone tener en cuenta un ciclo vital de la pareja, el cual pasa por el siguiente recorrido: 1) extraño-desconocido, que implica un contacto pero distante; 2) conocido, el contacto es más cercano; 3 ) amistad, comienza un intercambio íntimo; 4) atracción, existe un interés sexual del encuentro con el otro cuerpo; 5) pasión, los encuentros sexuales se hacen presentes; 6) romance, se hacen promesas y proyectos en común; 7) compromiso, se establecen lazos de confianza al cumplimiento de promesas y de los proyectos; 8) mantenimiento, el paso del tiempo y las rutinas edifican la relación; 9) conflicto, las discusiones sin posible resolución dañan la relación, y 10) alejamiento, destruye la relación, pues no hay interés en establecer los lazos de comunicación que una vez los había acercado. Sin embargo, para el pensamiento de tercer orden es necesario reconocer que no siempre presentan este orden dichos elementos; McDowell et al. (2018, 2019) indican que en cada fase hay que considerar la incertidumbre, que las experiencias de vida no están dadas y que éstas son meras descripciones de posibilidades, pero no son la realidad de las parejas.

Los(as) autores(as) coinciden en una terapia breve, cuya duración no sobrepase las 10 sesiones, distribuidas semanalmente, permitirán la elaboración de intervenciones que llaman estratégicas, en las que se tratará de evitar que el (la) consultante, pareja o familia, siga tratando de resolver sus dificultades mediante las mismas soluciones intentadas. La característica principal de estas intervenciones consiste en una hábil capacidad de maniobra del terapeuta cuya finalidad se enfoca en evitar confrontaciones que dificulten el desarrollo del proceso del cambio (Madanes, 2007). En este sentido, es evidente la influencia de las técnicas en hipnosis, que Milton Erickson desarrolló en su consulta privada.

\section{Correlaciones Teóricas}

Se utilizó como fuente directriz el pensamiento de tercer orden (McDowell et al., 2018, 2019), los ejes metodológicos de la terapia breve estratégica de Fisch et al. (2003), Madanes (2007) y Vicencio (2011), sin olvidar a Sternberg (2018) con las historias de amor, donde, como elementos particulares en el actuar del terapeuta familiar y estratégico, recordamos los siguientes aspectos.

\section{Conocer la historia de amor de la pareja}

Este momento de la terapia es el crucial, pues define los actores que influyen en la historia de la pareja, quiénes son la pareja, cuáles son los guiones de cada integrante de la pareja, así como la trama y las conclusiones de ésta (Sternberg, 1998).

Este supuesto coincide con la necesidad de no quedarse con definiciones vagas y huidizas en cada elemento del guión y de la trama de la historia de amor (Sternberg, 1998; Fisch et al., 2003). Lo que nos llevaría a conocer el incidente/trama que llevó a la pareja a buscar la consulta/otros guiones (Vicencio, 2011; Sternberg, 1998). En este primer contacto con la pareja es común encontrar los guiones que cada uno(a) tiene en tanto la queja como el reproche, así como el "guión oficial" para el terapeuta. Por lo que el (la) terapeuta debe abstenerse a ser colocado(a) en determinada posición en el guión de la pareja; es importante distanciarse de la definición inicial del problema, pues de lo contrario no podrá relacionarla con la historia de amor. En lugar de eso, el (la) terapeuta deberá modificar las definiciones del síntoma o la metáfora o el motivo de consulta expresada por la pareja; en consecuencia, el (la) terapeuta logrará modificar el sistema de interacciones de enfocarse en síntoma/ queja/motivo a pasar a la historia de amor (Madanes, 
2007). Con ello es necesario recordar que las historias de amor están enmarcadas en un sistema cultural falocentrista, heteronormado, clasista y racista, por lo que será importante explorar cómo los sistemas de opresión y subordinación han influido en las historias de amor de cada pareja (McDowell et al., 2018, 2019).

Es necesario preguntar acerca de la solución intentada por las parejas (Neuburger, 1998; McDowell et al., 2018, 2019): indagar respecto a las formas pautadas en que las parejas han intentado dar solución a sus problemas (Neuburger, 1998; Fisch et al., 2003; Madanes, 2007). A veces las parejas utilizan soluciones que Neuburger (1998) denomina "soluciones funcionales" y "soluciones problemáticas". Las soluciones funcionales las caracteriza como las que buscan la protección de la pareja ante las fugas de lo íntimo, lo que significa que consolidan su identidad, refuerzan sus fronteras que las separan del mundo exterior; Neuburger (1998) las identifica como riñas conyugales, las cuales en general terminan fortaleciéndolos en lo íntimo. De igual modo visualiza al "tenis conyugal" como un ritual en el que la pareja expresa en forma de intercambio agresividades que sólo pueden resolverse frenando el conflicto y citándose en lugares alternos para preservar el intercambio sano, equitativo e igualitario (McDowell et al., 2018, 2019).

Respecto a las soluciones problemáticas, Neuburger (1998) indica que los secretos, las mentiras y la desinformación pueden no sólo generar problemas en el aquí y el ahora, sino que, por lo contrario, atentan con la fundación de esta, entre ellos la infidelidad. Por otro lado, el autor considera que las soluciones disfuncionales son las que se caracterizan por hacer problemas de las soluciones, entre ellas los problemas de comunicación, la desinformación, que parecían resolverse conversando, se entraman problemas continuos.

Conocer estas soluciones ayuda para posibilitar (si el caso lo permite) la prescripción de paradojas, con el objetivo de producir la conducta sintomática, o la conducta expresada de modo metafórico por el síntoma. Para Madanes (2007), en las paradojas se prescribe al cónyuge sintomático una tarea penosa asociada con el síntoma o al no sintomático se le encarga una tarea penosa relacionada con el síntoma. Con ello se exagera paradójicamente la incongruencia del poder de la pareja mediante las directivas destinadas a instar a los cónyuges a reorganizarse en un poder más congruente y equitativo. Por ejemplo, que quien inicie el conflicto de pareja se dirija al baño, Ilevando de la mando al cónyuge; estando ahí, el que empezó el conflicto se quede de pie y el oyente se siente en la tasa de baño.
Conocer la propuesta de Neuburguer (1998) permite analizar la figura 1que nos sitúa en la configuración de la pareja y a entender la co-construcción del problema (Vicencio, 2011). Esto nos lleva de inmediato a conocer: 1) problemas que derivan del ciclo vital de la pareja y de la familia; 2) crisis de la pareja; 3) conflicto crónico; 4) dolores del cuerpo y del alma, y 5) dolores psicosociales. Para Vicencio (2011) estos elementos son los que darán a conocer el mapa inicial para el trabajo de terapia de pareja y, enriquecidos con el pensamiento de tercer orden, permite pensar en los sistemas culturales que dan origen a cada una de las situaciones anteriores, posibilita una comprensión contextual y de justicia social en la que la pareja se ha visto envuelta (McDowel et al., 2018, 2019).

Para Fisch et al. (2003) es importante decidir qué se debe evitar: En este caso la maniobra del terapeuta es indispensable para no lograr participar en el mantenimiento del síntoma (Fisch et al., 2003; Madanes, 2007).

\section{Formular un enfoque estratégico}

Es fundamental tener un método que refleje un pensamiento estratégico; por ejemplo: 1) escucha activa; 2) validación; 3) preguntas circulares; 4) síntesis de la información; 5) validación de hipótesis; 6) elaboración de tareas, y 7) seguimiento. Mediante las distintas posturas que adopta el terapeuta en sesión (como cercana, intermedia o distante), éste obtiene información suficiente para recurrir a los cambios deseados en la vida de la persona (Fisch et al., 2003; Madanes; 2007).

\section{Formular tácticas concretas}

Lo más importante del siguiente tema es que el terapeuta no se limitará a sólo esperar a que la pareja deje de hacer algunas cosas por otras, sino que en vez de eso construirá la sesión posterior en un diálogo donde por medio de determinado tipo de preguntas logre "dar al clavo" con algunas modificaciones en la forma en crear la conducta problema, entendiendo así que dichas conductas están cristalizadas y pautadas en un ritual, que la gente las viene haciendo desde hace ya bastante tiempo (Fisch et al., 2003; Vicencio, 2011).

En este sentido, el (la) terapeuta podría solicitar a la pareja que haga un pequeño cambio en el manejo del problema, dando como resultado un impacto importante en la forma de establecer el problema. Se fomenta la unión de la pareja y el mejoramiento de su relación mutua, para que ya no recurrieran al sistema de interacción enfocado en el síntoma como una analogía y una solución de las dificultades (Fisch et al., 2003; Madanes, 2007). 


\section{Encuadrar la sugerencia en un contexto:} Vender la tarea

El sentido práctico de este apartado, la sugerencia de vender una tarea, conlleva uno de los elementos más importantes dentro de la terapia, y esto es la realización de las tareas. Aunque el hecho de que no hagan las tareas también proporciona información al terapeuta, el sentido de este enfoque y todos en general es que la pareja comienza a hacer de manera espontánea cambios, pero es necesario que, dentro del repertorio de habilidades del terapeuta, se encuentre la forma de influir de modo positivo en la pareja, para que ésta haga las tareas sugeridas (Madanes, 2007).

\section{Formular objetivos y evaluar el resultado}

Una de las maneras en que los autores retoman el modo de elaborar los objetivos y la evaluación de los resultados, tanto esperados como inesperados, es por medio de algunas formas en que el (la) terapeuta pregunta por un informe de los resultados obtenidos en las intervenciones o en las tareas sugeridas; de igual modo, el (la) terapeuta ha de basarse en su propia estimación acerca del impulso de la pareja respecto a su situación problemática (Madanes, 2007).

\section{Caso Anna y Dan}

La consolidación de las historias de amor en las relaciones que presenta Nichols (2004), varían según la fluctuación de tres componentes (amor, pasión y compromiso) entre los actores, los guiones y las tramas.

En esta película los actores que pretenden ser absolutamente sinceros y se comunican directamente sus infidelidades como parte de sus tramas. En un juego a cuatro bandas, dos parejas intercambian pasiones, deseos y sentimientos. El guión por el cual se han movido los actores es : El dermatólogo Larry, de 34 años, es un hombre blanco cis $^{1}$, heterosexual y de clase media con estudios universitarios, se muestra como un individuo que antepone la pasión por encima de todas las consideraciones. La fotógrafa Anna, una mujer blanca, cis, heterosexual, de clase media y con estudios universitarios, se muestra como una mujer débil y dependiente, que se revelaría como una mujer que manifiesta una necesidad clara y directa de formalizar sus relaciones para sentirse segura y amada. Dan, de 34 años, es un hombre blan$\mathrm{co}$, cis, heterosexual, clase media y universitario, escritor de obituarios, se muestra como una persona cínica y manipuladora, que va de ida y vuelta en sus relaciones sin comprometerse, para al final encontrarse en una relación con Anna. Por su parte, Alice es una mujer, cis, heterosexual, joven, de 20 años, migrante, nudista, que también se muestra como una mujer dependiente, pero que sabe cómo defenderse ante las traiciones y manipulaciones de Dan y Larry, así como de los hombres en general, pues su oficio le ha mostrado algunas claves emocionales para cuidarse del machismo.

El inicio del entramado, el sistema relacional, comienza con Anna y Dan, quienes tuvieron un acercamiento característico del "amor insensato" (Díaz-Loving, 2005; Vicencio, 2011) por ser un amor pasional, donde había intimidad, pero no compromiso. Anna, al saber del compromiso de Dan con Alice decide alejarse. Para provocar a Anna, Dan decide incitar a Larry para que conociera a Anna. Anna, para alejar a Dan, elige comprometerse con Larry, donde la relación muestra poca pasión e intimidad; sólo existía el compromiso. Con el paso del tiempo las redes sociales de las parejas se entrelazan y complejizan, como la relación fuera del matrimonio de Larry con Alice, un amor pasional provocado por la infidelidad de Anna con Dan.

El contexto social de las relaciones desiguales entre mujeres y hombres, como se puede observar en el análisis anterior, ese "amor insensato", cobra sentido con base al género, donde los hombres eligen la intimidad pero no el compromiso, por lo que una de las hipótesis de trabajo puede estar dirigida a comprender como se configuró esa relación entre Anna y Dan, en donde Anna se encuentra en desigualdad de condiciones respecto a Dan, ¿cómo aprendió en su vida que el lugar de las mujeres es seguir a los hombres? (McDowell et al., 2018, 2019).

Caso parecido en la relación de Larry con Alice, en el que se describió como amor pasional, sin embargo en el contexto del tráfico de mujeres, la precarización de la vida las conduce a empleos como el que Alice ejerce para sobrevivir por su estatus de migrante. Larry llega al lugar de trabajo de Alice con intenciones obvias de consumo sexual. En la construcción de la identidad masculina, el consumo de pornografía, así como de prostitución, se convierte en un "pacto" común entre hombres con tal de reafirmar su masculinidad y no poner en duda su heterosexualidad, por lo que se puede comprender la reacción de celos y control de Larry hacia Alice cuando él no puede controlarla (McDowell et al., 2018, 2019).

La relación entre Dan y Larry, la cual expresa rivalidad masculina, en la que Dan provoca a Larry para

1 El término "cis" hace referencia a las personas que aceptan el género que se les asignó al nacer, contrario a las personas trans, quienes en sus trayectorias de vida descubren que no se encuentran cómodas con el género que se les asignó al nacer; es pertinente hacer estos señalamientos en la presentación de casos clínicos, pues da a conocer que, así como hay personas que se sienten a gusto con el género que se les asignó al nacer, hay otras personas que no, y es parte de la identidad de las personas (Gómez-Lamont y Silva, 2019). 
que conozca a Ana, lo que puede conducir a la idea de que las mujeres son ese objeto de discordia entre hombres, Ilevándonos a la idea que el análisis de género no es exclusivo de hombres con mujeres, sino de hombres con hombres y mujeres con mujeres; en ese tenor es importante tener éstas hipótesis con base en el género que performan cada uno de los integrantes de estas historias (McDowell et al., 2018, 2019).

En la relación de Ana y Dan, el "juego" de esta pareja se podría describir por medio de los celos pasionales de Dan hacia Larry por haberse casado con Anna. Larry, al mostrar sus celos obsesivos por Anna, decide serle infiel a Anna con Alice. La insatisfacción marital de Anna con Larry causa que Anna decida tener una "aventura" con Dan. La infidelidad de Anna ocasiona que Larry busque a Alice provocando el divorcio de Anna con Larry. Para vengarse de Dan, Alice decide ser infiel a Dan con Larry. Para Gottman (1994) estas parejas pudieran representar los cuatro jinetes del Apocalipsis, de acuerdo con los datos presentados entre Larry y Ana, Dan y Alice y luego Anna y Dan. Este recuento de los daños, desde el pensamiento de tercer orden (McDowell et al., 2018, 2019), ayuda a recordar que toda conducta comunica género, en estos casos comunica cómo de modo cultural se aprenden las relaciones entre hombres y mujeres: relaciones de desigualdad de condiciones (Anna y Dan, Alice y Larry), relaciones de poder y dominación (Dan y Larry) y rivalidad y competencia en pro de tener a un hombre (Anna y Alice). Estas observaciones son hipótesis de trabajo importantes para la elaboración de intervenciones específicas en la terapia estratégica, y dejamos de mirar un "juego" donde supuestamente se tiene una igualdad de condiciones, por conocimiento de jugadas, tablero y piezas, para ver una distribución desigual de las reglas de juego entre los géneros (figura 1).

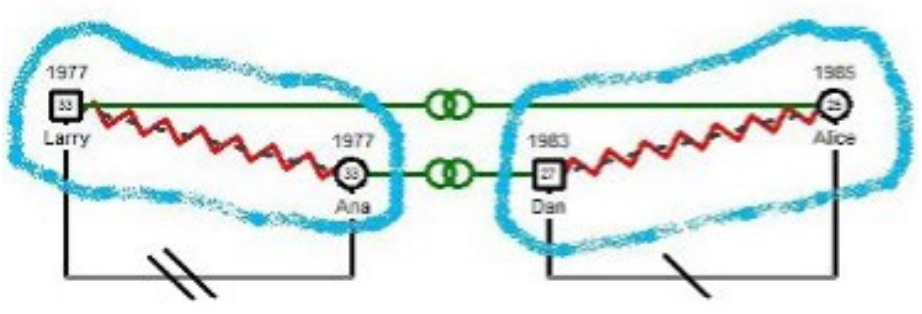

Figura 1.

Circuito de interacción de las parejas (duograma² de las parejas).
El motivo de consulta y el guión de Anna fue el hecho de que pese a haber aceptado su carga de responsabilidad en cuanto a su infidelidad con Dan, la cual le causó el divorcio con Larry. Anna afirma haber hecho cualquier cosa para dejar de pelear con Dan respecto a su infidelidad con Alice, pero confiesa no lograr dejar de pelear.

Su problema era un enfrentamiento incontrolable: por lo que cuenta Anna, eran capaces de pelear varias veces al día para luego hacer las paces mediante relaciones sexuales, donde al término de éstas se prometían tolerancia, para después recaer en nuevas peleas provocadas por la misma circunstancia: el resentimiento mutuo.

Su solución intentada fue que a menudo, por la noche, después de tener relaciones sexuales, aprovechaban la dulzura de esa atmósfera, hablaban horas y explicaban con serenidad los motivos de su enfado, Ilegando incluso, la mayoría de las veces, a la recíproca comprensión (lo que recuerda las soluciones funcionales de Neuburguer, 1998). Sin embargo, al día siguiente, a la mínima discordancia o dificultad cotidiana peleaban de nuevo de manera rabiosa - cuando la solución se vuelve un problema- (Neuburguer, 1998). El objetivo desarrollado en conjunto con Anna era que ella se daría cuenta de que iba por un buen camino, cuando su resentimiento hacia Dan no se hiciera presente y lo notaría cuando no le contestara de forma brusca y grosera. Pero para que una terapia sea de tercer orden, es importante integrar a los hombres en la carga de responsabilidad de la situación; Anna reacciona de esa forma por las enseñanzas culturales de cómo las mujeres se relacionan con los hombres, haciendo que el cambio y la permanencia en la relación recaigan en ella, por lo que es importante incluir a Dan en el compromiso del cambio, en modificar sus actitudes y reconocer su parte del daño, y que el incentivar la resolución de los conflictos con el sexo es más una resolución problemática y masculina que equitativa (McDowell et al., 2018, 2019).

La estrategia fue la siguiente.

En resumen, en la fase de evaluación que el (la) terapeuta ha de efectuar en la primera entrevista, consistirá en obtener una definición del problema que trae a consulta a la familia, la pareja o a la persona, así como de las soluciones intentadas en términos de conductas concretas. Para el caso presentado, Madanes (2007), Vicencio (2011), Fisch et al. (2003) y McDowell et al. (2018, 2019) proponen preguntar: “¿Cuál es el problema que les trae aquí?"; cuando el problema parece una dificultad normal recomiendan inquirir: "¿Cómo se con-

2 El duograma es una herramienta que permite la elaboración de mapas relaciónales en las historias de las parejas; en el caso presentado indica edades, cruces conflictivos y cruces afectivos; se puede ver que existen estas dinámicas entre Anna, Dan, Larry y Alice (Gómez-Lamont y Silva, 2019). 
vierte en problema la situación que ustedes me comentan?"; así, si la conducta sintomática viene produciéndose desde hacía ya varios años se pudiese preguntar los siguiente: "¿Qué les ha llevado a consultar por el problema ahora?"; una vez completada dicha fase, es necesario considerar una definición operativa del problema, así como de la indagación de las soluciones intentadas. En el trabajo o la maniobra que el (la) terapeuta ha de efectuar, es lograr que los pacientes lleguen a la concreción del motivo de consulta que los lleva a pedir terapia, para lo cual Madanes (2007), Vicencio (2011) y Fisch et al. (2003) recomiendan y dejan un tiempo completo en la adopción de una posición de inferioridad, a la cual pueda llevar al (la) terapeuta a conseguir mayor información acerca del problema. Por tanto, el efecto puede ser ventajoso en el sentido de enganche terapéutico, formación de la colaboración y demás estrategias para la permanencia terapéutica.

Para lograr la equidad, McDowell et al. (2018, 2019) proponen una serie de intervenciones basadas en interrogatorio circular que pudieran funcionar para que Dan visualice su dominio y Anna se dé cuenta del nivel de desventaja en que se encuentra en relación con Dan, para que Dan se dé cuenta de su poder y dominio y sea responsable de sus actos.

El terapeuta puede iniciar la técnica indicando que leerá una lista de derechos en una relación de pareja; ellos escucharán con atención, y por cada derecho que sientan que tienen, tomarán una bola de unicel; si sienten que no tienen el derecho, no toman bola de unicel; al finalizar la lista contarán los derechos que cada uno(a) percibe que tiene en la relación, reflexionarán acerca del origen de esos derechos, las injusticias y las posibles soluciones eficaces ante esta situación.

Lista de derechos en una relación de pareja

1. Tengo derecho a expresar libremente mis emociones sin sentir que de hacerlo enojaré más a mi pareja.

2. Tengo derecho a ver a mis amistades libremente sin que mi pareja me cuestione por ello.

3. Tengo derecho a expresar mi pensamiento sin que mi pareja considere que mi mente está equivocada o influenciada por alguien o algo.

4. Tengo derecho a vestirme como quiera sin que mi pareja me critique o considere que deba cambiarme por ser muy provocativa.

5. Tengo el derecho a que mi pareja no alce la voz cuando algo no le parezca

6. Tengo derecho a que mi pareja no controle mis mensajes y llamadas, a que no revise mi celular.
7. Tengo derecho a no sentirme asustado(a) de mi pareja cuando no esté de acuerdo con sus gustos o planes de fin de semana.

8. Tengo derecho a controlar mis gastos como yo quiera y decida.

9. Tengo derecho a expresar libremente mi satisfacción sexual sin sentir que le ofenderé.

10. Tengo derecho a expresar libremente a mi pareja cuando no quiera tener relaciones sexuales sin sentir que le ofenderé.

Como se puede observar, esta tarea que es directa debe de ser "vendida" de modo que la pareja sienta confianza de ser sincera de reconocer los puntos en que no siente igualdad de condiciones con tal de reconocer los lados fuertes de su relación, así como las injusticias dentro de la misma (McDowell et al., 2018, 2019).

\section{ANÁLISIS Y DISCUSIÓN}

Para comenzar a analizar las consideraciones éticas de este modelo es necesario recordar que las terapias en el campo de la Psicología no deben verse como un mero sistema de técnicas para el cambio de la conducta, sino deberán reflexionar y visibilizar el sistema de creencias en cuanto al ser humano que representan con sus intervenciones, sus creencias y valores en cuanto a lo normal y lo aceptable.

Las terapias breves, así como las estratégicas, deberán reconocer su enfoque utilitario y contractual, el cual, en su base moral, buscan maximizar el placer a la mayor cantidad de personas y reducir el dolor en corto tiempo en una relación pactada entre terapeuta y consultante (Gómez-Lamont, 2016). Para el pensamiento de tercer orden es fundamental reconocer los dilemas éticos que puedan estar presentes en la psicoterapia, como el utilitarismo tiene por propiedad buscar beneficiar al mayor número de personas; sin embargo, el dilema que presenta es sopesar lo que la mayoría quiera sobre las minorías, en terapia se pudiera traducir en las siguientes omisiones, por ejemplo, de forma individual, darle peso a "la mayoría de los problemas" sin mirar temas que parecieran "menores o irrelevantes", como darle mayor cabida a los temas del motivo de consulta o al "guión oficial", sin mirar temas como la afectación subjetiva e indirecta que tiene el clasismo respecto al motivo de consulta; en caso de temas de una pareja heterosexual, privilegiar el guión de la mayoría de los varones en la consulta (pudiera ser el terapeuta varón y el varón de la pareja) respecto al guion de la mujer. En ese tenor, desde el pensamiento de tercer orden una forma de resolver ese dilema ético en terapia pudiera usarse la equidad como método de redistribución en la terapia, es decir, posicionarse desde la parcialidad y 
la igualdad, brindar a todos(as) el mismo espacio para expresar ideas, sin concentrar la atención a una sola persona; de esta manera pudiera contribuir a una mejor forma de trabajo terapéutico (Gómez-Lamont, 2016).

\section{ConcLusiones}

Si bien es cierto que la pareja se puede ver como un grupo y recordar que se conforma de un arreglo ficticio y litúrgico en el que, en la mayoría de los casos, la fidelidad será un acuerdo implícito como compromiso con la pareja. Por tanto, pertenecer a un grupo implica demostrar una creencia en los mitos y en las ficciones del grupo de pertenencia, así como la participación de los mismos en los rituales de la familia de origen de cada uno de los miembros de la pareja.

En el caso presentado, la infidelidad podría tratarse como un tema de acuerdos implícitos de la pareja, donde la demanda de Ana estaba estrechamente relacionada con el intento de resolver los conflictos por medio de peleas en torno a la infidelidad mutua, por lo que interferir en el "circuito" de soluciones de la pareja fue algo indispensable. Teniendo en cuenta que el tipo de intervenciones paradójicas son eficaces y eficientes, si antes se ha tenido un manejo por parte del terapeuta, donde ha persuadido de modo excelente a la pareja para que ésta compre la tarea e intente algo similar a lo que vienen haciendo, pero con un cambio introducido sagazmente.

Por tanto, tener presente el tema de la infidelidad es de importancia, siempre y cuando se comprenda el proceso en el que se dio y las consecuencias que dejó, por lo que la toma de decisiones no deberá enfocarse en la búsqueda de los culpables, sino en la comprensión del cómo surgió esta dificultad que puede alentar los recursos y cohesionar a la pareja, o destruirla por completo, donde los mitos de cada uno de los miembros de la pareja estarán presentes en todo momento.

Las limitaciones en este modelo son diversas; en primer lugar el trabajo con la categoría analítica de "patrones" se ve reducida cuando a ésta se le añade el planteamiento del pensamiento del tercer orden (Mcdowell et al., 2019), pues al trabajar con los "mapas del amor" se incluye una mirada más compleja en la que se abordan diversos elementos socioculturales en los modelos de terapia que, a diferencia de la escuela del MRI, nos enfocamos en una sola manera de mirar la realidad. Por otro lado, con las dimensiones éticas comentadas en el último apartado, el cual reconoce a la terapia breve como utilitaria y consecuencialista, en el que ve al poder y al engaño como medios para lograr un fin. El placer o el beneficio deberán ser motivo de reflexión, el cual se logrará si, y sólo si, el (la) terapeuta usa técnicas que motiven la equidad y la redistribución de recursos materiales y psicológicos en las parejas cis-heterosexuales, cis-homosexuales o trans-hetero, homo, bi o pan sexuales.

\section{Referencias}

Asen, E., \& Jones, E. (2000). Terapia sistémica de pareja y depresión. Bilbao: Desclée de Brouwer.

Baizán, B. (2007). El paso de la muerte en la pareja: De la fidelidad a la infidelidad. En L. L. Eguiluz (comp.), Entendiendo a la pareja: Marcos teóricos para el trabajo terapéutico ( $p p$. 143-167). México: Pax.

Butler, C. (2015). Intersectionality in Family Therapy Training: Inviting Students to embrace the complexities of lived experience. Journal of Family Therapy, 37(4), 583-589. https://doi.org/10.1111/1467-6427.12090

Collins, S., \& Arthur, N. (2010). Culture-infused counselling: A model for developing multicultural competence. Counselling Psychology Quarterly, 23(2), 217-233. https:// doi.org/10.1080/09515071003798212

Díaz-Loving, R. (2005). La Psicología del amor: Una visión integral de la relación de pareja. México: Miguel Ángel Porrúa/UNAM.

Fisch, R., Weakland, J. H., \& Segal, L. (2003) La táctica del cambio, cómo abreviar la terapia (pp. 19-30). Barcelona: Herder.

Gómez-Lamont, M. (2016). Bioética, feminismo y la dignidad de las "mujeres": Proceso social y cambio de valores en las terapias familiares. Alternativas en Psicología. No. 36.

Gómez-Lamont, M., \& Silva, A. (2019). La terapia familiar sistémica y la comunidad LGBT +. México: UNAM/PAX/ LEED.

Gottman, J. M. (1994). What Predicts Divorce?: The relationship between marital processes and marital outcomes. Hilsde, N. J: Lawrence Erlbaum Associates.

Gottman, J. M., \& Silver, N. (1994). Why Marriages Succeed or Fail: What you can learn from the breakthrough research to make your marriage last. Nueva York: Simon y Schuster.

Gutiérrez, D. (2018). The role of intersectionality in marriage and family therapy multicultural supervision. The American Journal of Family Therapy, 46(1), 14-25. https://doi.org/1 $0.1080 / 01926187.2018 .1437573$

Madanes, C. (2007). Terapia familiar estratégica. ArgentinaEspaña: Amorrortu.

McDowell, T., Knudson-Martin, C., \& Bermúdez, M. (2019). ThirdOrder Thinking in Family Therapy: Addressing Social Justice Across Family Therapy Practice. Family Process. 58(1), 9-21. https://doi.org/10.1111/famp.12383

McDowell, T., Kudson-Martin, C., \& Bermudez, M. (2018). Socioculturally Attuned Family Therapy: Guidelines for Equitable Theory and Practice. Routledge: USA. Springer.

Neuburger, R. (1995). La familia dolorosa. Mitos y terapias familiares. Barcelona: Herder.

Neuburger, R. (1998). Nuevas parejas. Buenos Aires: Paidós. Nichols, M. (Director \& Productor) (2004). Closer, llevados por el deseo (película). USA: Columbia Pictures. 


\section{Meta-Análisis del Artículo}




\section{Dimensión Cuantitativa}

\section{Perfil de Evaluación entre pares}
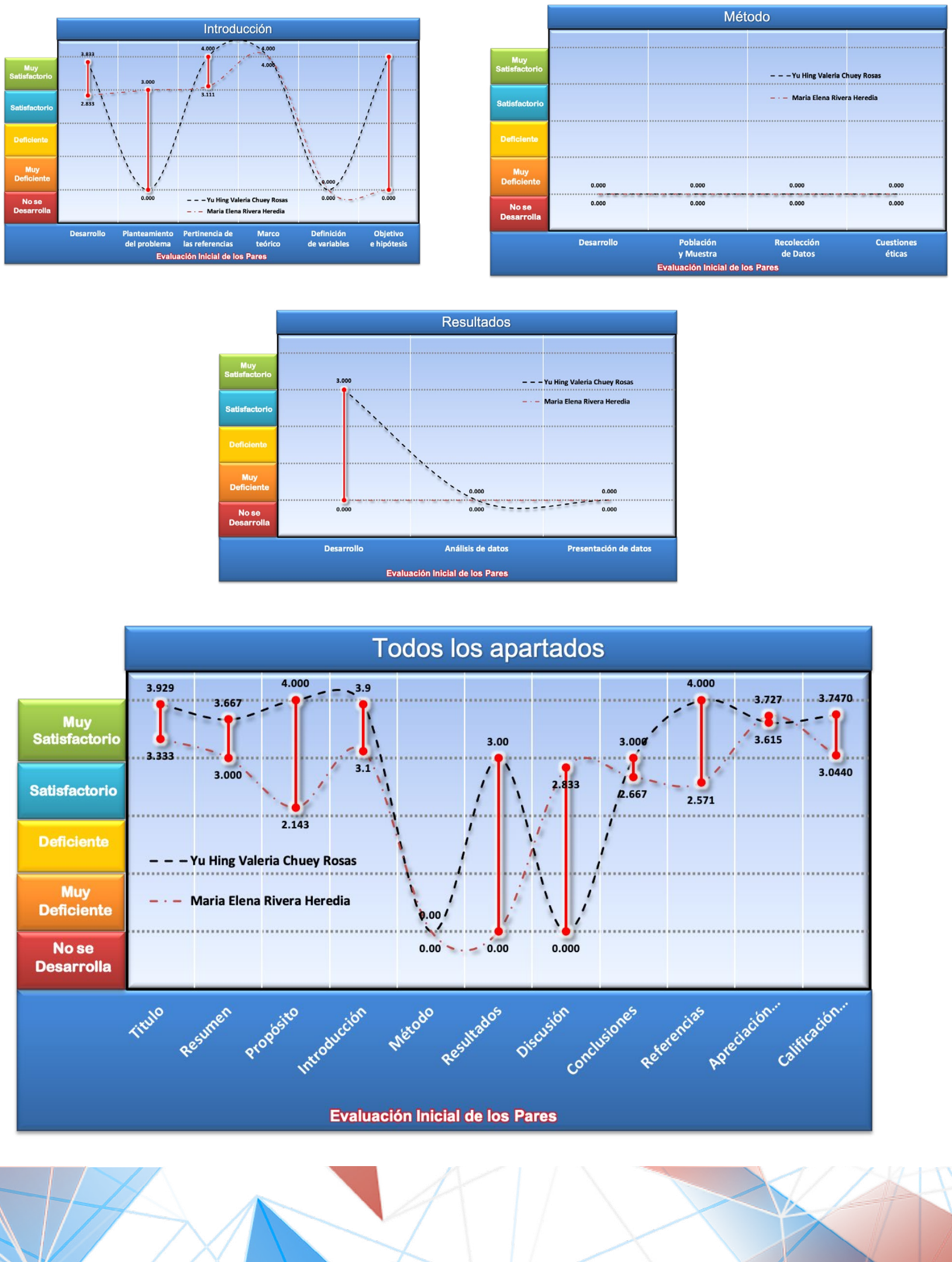


\section{Índice de Concordancia}

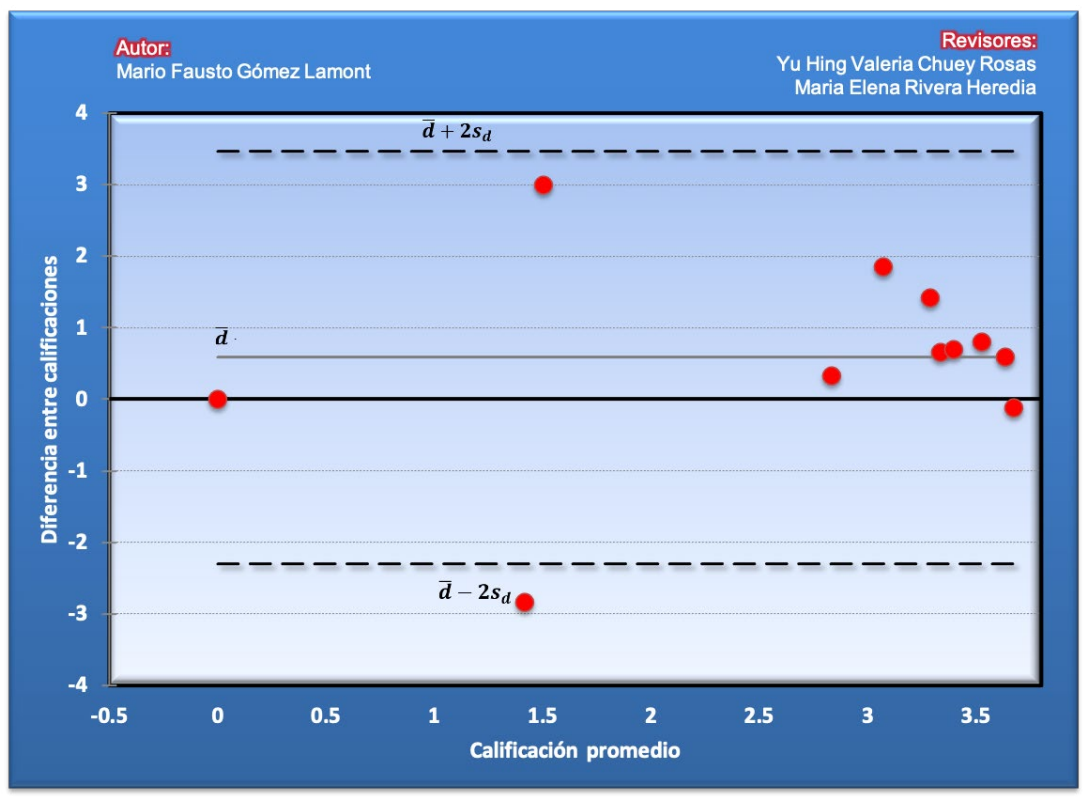

Índice de Acuerdo

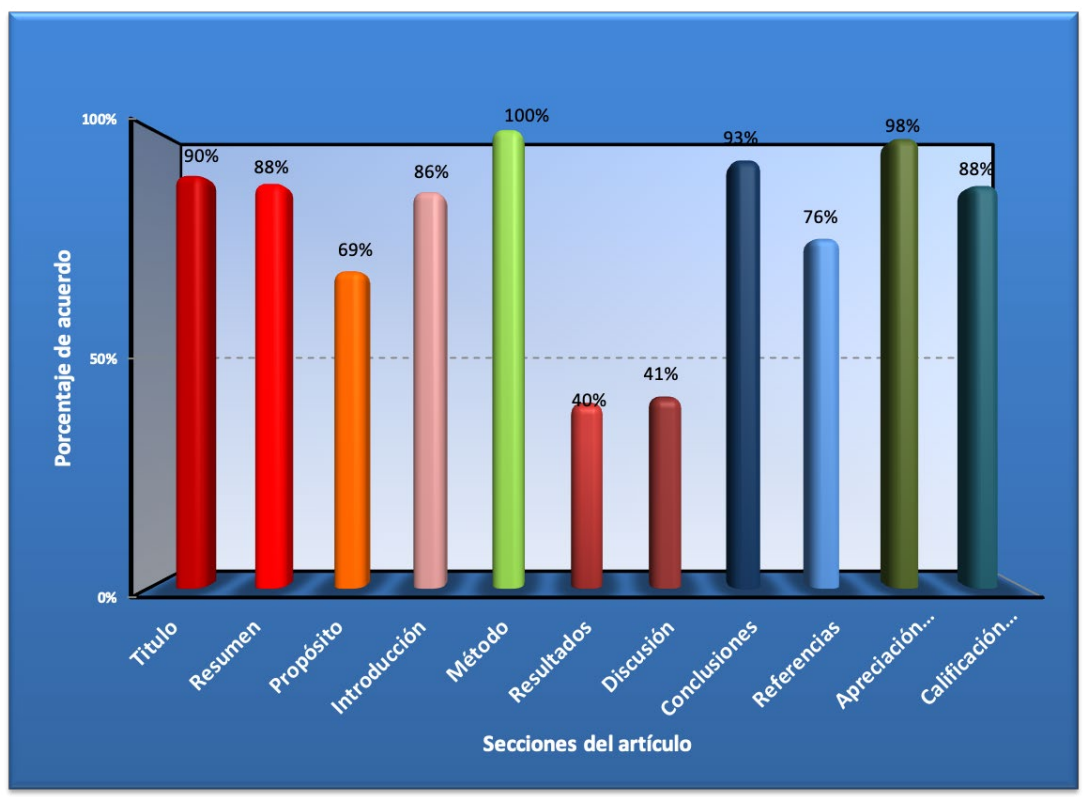




\begin{tabular}{|c|c|}
\hline Revisor 1 & Revisor 2 \\
\hline Yu Hing Valeria Chuey Rosas & Maria Elena Rivera Heredia \\
\hline \multicolumn{2}{|c|}{ Título/Autoría } \\
\hline $\begin{array}{l}\text { Sugerencia: Omitir el "de" después de Terapia, probable- } \\
\text { mente fue un error al escribirlo: El Pensamiento de Tercer } \\
\text { Orden en la Terapia -de- Breve Estratégica para Parejas } \\
\text { que vivieron una infidelidad }\end{array}$ & $\begin{array}{l}\text { El titulo tiene } 18 \text { palabras y se sugieren } 15 \text {. Es posible re } \\
\text { ducirlo. Eliminando "El" al inicio del titulo y reduciend } \\
\text { las últimas palabras a "en parejas con infidelidad" }\end{array}$ \\
\hline
\end{tabular}

\section{Resumen}

Sugerencia: se podría incluir al inicio cuál es el objetivo $\quad$ Sugiero incorporar en el resumen que en el artículo se del escrito, que fue el análisis conceptual del modelo analiza una película sobre parejas que viven infidelidad. estratégico para el trabajo con parejas heterosexuales. El resumen contiene 184 palabras. Es necesario reducirlo. Queda anotado más adelante, pero es una sugerencia Pueden incorporar 2 palabras clave adicionales pues solo que pueda ir señalado antes para tener mayor claridad. se introdujeron 3

\section{Próposito del Estudio}

Así como en el resumen, podría resaltarse el propósito del escrito después de la introducción.

El propósito del estudio se da a entender a lo largo del documento pero no se clarifica, por lo que sugiero dar un espacio especial al propósito del estudio.

\section{Introducción}

Los elementos que aquí se evalúan no son adecuados para dictaminar el artículo dado que se trata de un artículo de revisión de literatura y aplicación de la misma a un caso

La introducción es adecuada, hacia el final se señalan los objetivos, tal vez solo después de este enunciado: "con tal de fundamentar la necesidad de integrar el pensamiento de tercer orden a éste tipo de terapia”, se podría enriquecer al idea. hipotético derivado de una película. Sin embargo, es relevante en cuanto a que clarifica la forma de trabajo y principios de una forma de tratamiento desde los enfoques sistémicos y posmodernos que se puede aplicar a la atención de un frecuente problema conyugal: la infidelidad. Considero que puede ser un artículo de interés para los lectores de la revista, siempre y cuando se considere que corresponde a la línea editorial que esta maneja. El documento revisado sería un muy interesante capítulo de libro.

Método

No aplica en esta sección, no hay ningún comentario ni sugerencia.

Ninguno de los rubros de estas secciones pueden aplicarse para el análisis del artículo que se entregó a revisión. El que no los cubra no quita el valor de dicho documento, sino que hace referencia a que se trata de una obra de otra naturaleza. 


\begin{tabular}{|c|c|}
\hline Revisor 1 & Revisor 2 \\
\hline \multicolumn{2}{|c|}{ Resultados } \\
\hline $\begin{array}{l}\text { No aplica en esta sección, no hay comentarios o suge- } \\
\text { rencias. }\end{array}$ & Se trata de un artículo teórico y no empírico. \\
\hline \multicolumn{2}{|c|}{ Discusión } \\
\hline Sugerencia: revisar el uso de comas y corregir una palabra. & $\begin{array}{l}\text { Si presenta un apartado de discusión en torno a los ar- } \\
\text { gumentos que fue exponiendo a lo largo del documento. } \\
\text { El análisis que incluyó me parece adecuado, pero breve. } \\
\text { Puede profundizarlo y ampliarlo más. }\end{array}$ \\
\hline \multicolumn{2}{|c|}{ Conclusiones } \\
\hline $\begin{array}{l}\text { Se podría resaltar las propuestas de investigaciones futu- } \\
\text { ras respecto al tema, se menciona en el escrito, pero en } \\
\text { esta sección podría señalarse aún más. }\end{array}$ & $\begin{array}{l}\text { Pese a lo interesante del tema, y a la utilidad práctica de este } \\
\text { tipo de información teórica, para mí una de las principales } \\
\text { limitaciones del artículo es que las fuentes de referencia } \\
\text { no son recientes. Considero que de no existir recientes, } \\
\text { seria un punto de gran interés incluir en las conclusiones } \\
\text { el porqué no se continuó abonando conocimiento a esta } \\
\text { modalidad de propuesta terapéutica, el señalar qué ha pa- } \\
\text { sado con la terapia breve estratégica al paso del tiempo, así } \\
\text { como la influencia del pensamiento de Tercer Orden. }\end{array}$ \\
\hline \multicolumn{2}{|c|}{ Referencias } \\
\hline Revisar espacios de más en las referencias. & $\begin{array}{l}\text { Solo cuenta con } 19 \text { referencias. Entre ellas solo } 5 \text { corres- } \\
\text { ponden a los últimos } 5 \text { años. Incluye referencias clásicas } \\
\text { del tema que requieren ser conservadas, sin embargo, con- } \\
\text { sidero necesario ampliar las fuentes de referencia y actua- } \\
\text { lizar la información sobre lo que ha pasado con la terapia } \\
\text { breve estratégica desde su fundación a la fecha. También } \\
\text { sugiero se incorporen resultados de investigaciones sobre } \\
\text { factores asociados con la infidelidad y sobre enfoques y } \\
\text { técnicas terapéuticas que se han utilizado para abordarla, } \\
\text { u otras formas de pensamiento que puedan estar ligadas } \\
\text { al pensamiento del tercer orden, en el tratamiento con pa- } \\
\text { rejas como pueden ser las propuestas de investigación de } \\
\text { tipo transformativo o emancipatorias. }\end{array}$ \\
\hline
\end{tabular}


